\title{
Simulation and optimization of VRPSA system based on pseudo-transient method
}

\author{
Zhaoyang Niu ${ }^{1}$, Wenbin $\mathrm{Li}^{1}$, Zhongli Tang${ }^{2}$, Yuanhui Shen ${ }^{1}$, and Donghui Zhang ${ }^{1}$ \\ ${ }^{1}$ Tianjin University \\ ${ }^{2}$ Affiliation not available
}

November 18, 2021

\begin{abstract}
In order to address the challenge of pressure swing adsorption system optimization, an optimization framework based on pseudo transient continuation method was used and vacuum rapid pressure swing adsorption process for oxygen production as a typical example. A pseudo transient model library was established and a robust two-stage dynamic tearing model was proposed to deal with the cyclic steady state conditions. Furthermore, the time constants were discussed and a practical time constant strategy and expressions were proposed for the stability and efficiency of calculation. Finally, reduced successive quadratic programming and time relaxation algorithm were used for the optimization of the two systems respectively, and the optimization results showed that although the simulation time of pseudo transient system is slightly higher than that of single discretization system, the optimization expense of single discretization system in two cases were 5.7 times and 11.6 times of that of pseudo transient system respectively.
\end{abstract}

\section{Hosted file}

manuscript.docx available at https://authorea.com/users/446924/articles/546039-simulationand-optimization-of-vrpsa-system-based-on-pseudo-transient-method 